Bundesgesundheitsbl 2009 $\cdot 52: 745-752$

DOI 10.1007/s00103-009-0876-7

Online publiziert: 12. Juni 2009

(c) Springer-Verlag 2009

\author{
S. Kohler · A. Richter - T. Lampert · G.B.M. Mensink \\ Abteilung für Epidemiologie und Gesundheitsberichterstattung, \\ Robert Koch-Institut, Berlin
}

\title{
Alkoholkonsum bei Jugendlichen in Deutschland
}

\section{Ergebnisse aus EsKiMo}

Ein regelmäßiger Alkoholkonsum ist unter Jugendlichen in Deutschland weit verbreitet und somit ein bedeutendes Gesundheitsrisiko. Insbesondere das häufig geringe Alter beim Erstkonsum ist besorgniserregend [1]. Es wurde ein Zusammenhang zwischen geringem Alter beim Erstkonsum alkoholischer Getränke und einem erhöhten Risiko für stressbedingte und pathologische Konsummuster bis hin zu einer Abhängigkeit im späteren Lebensverlauf beobachtet $[2,3]$. Kinder und Jugendliche sind bezüglich der mit dem Alkoholkonsum verbundenen Gesundheitsrisiken als besonders vulnerable Gruppe anzusehen. So kann die Entwicklung des Gehirns und des Nervensystems durch Alkoholintoxikationen nachhaltig beeinträchtigt werden [4]. Laut der WHO-Jugendgesundheitsstudie „Health Behaviour in School-aged Children (HBSC)“ tendieren Jugendliche mit einem problematischen Alkoholkonsum häufiger zu psychosomatischen Beschwerden und einer geringeren gesundheitsbezogenen Lebensqualität [5]. Als besonders riskant ist das sogenannte „Binge Drinking “ einzustufen. Eine international einheitliche Definition für dieses Konsummuster gibt es nicht; ein derzeit häufig verwendetes Kriterium ist der Konsum von fünf oder mehr Gläsern Alkohol zu einer Trinkgelegenheit $[6,7]$. Die Trendanalysen des Epidemiologischen Suchtsurveys (ESA) aus dem Jahr 2006 zeigen, dass das Binge Drinking unter jungen Erwach- senen in den letzten Jahren deutlich zugenommen hat [8]. Entsprechende Zahlen für Jugendliche aus Untersuchungen der Bundeszentrale für gesundheitliche Aufklärung (BZgA) schwanken zwar von Jahr zu Jahr, zeigen aber ebenso besorgniserregende Prävalenzen $[7,9]$.

Im Jahr 2006 mussten in Deutschland 24.380 Kinder, Jugendliche und junge Erwachsene im Alter von 10 bis 20 Jahren aufgrund ihres Alkoholkonsums im Krankenhaus behandelt werden; im Jahr 2003 war es noch ein Drittel weniger [10]. In diesem Zusammenhang sind neben der erhöhten Morbidität, Mortalität und den gesellschaftlichen Kosten auch die enormen sozialen und immateriellen Konsequenzen (zum Beispiel Gewalt, Familienprobleme, Probleme am Arbeitsplatz) zu berücksichtigen. Um die Probleme, die mit übermäßigem Alkoholkonsum bei Jugendlichen einhergehen, zielgruppenspezifisch bewältigen zu können, ist neben einer regelmäßigen Bestandsaufnahme die Identifikation von Risikogruppen wichtig. Ziel dieser Auswertungen ist es, mit Daten aus EsKiMo (Ernährungsstudie als KiGGS-Modul), die aktuelle Verbreitung des Alkoholkonsums bei Jugendlichen zu untersuchen. Neben alters- und geschlechtsspezifischen Unterschieden werden die Wohnregion, der Sozialstatus, der Migrationshintergrund und der von den Jugendlichen besuchte Schultyp als mögliche Differenzierungsmerkmale betrachtet.

\section{Methode}

EsKiMo wurde als Nachfolgemodul des Kinder- und Jugendgesundheitssurvey (KiGGS) durchgeführt. Für KiGGS wurden im Zeitraum von Mai 2003 bis Mai 2006 umfangreiche Daten zur körperlichen und psychischen Gesundheit von 17.641 Kindern und Jugendlichen im Alter von o bis 17 Jahren erhoben. Hierzu wurden 167 Untersuchungsorte (Sample Points) proportional nach Bundesland und Gemeindegröße in Deutschland ausgewählt. Innerhalb dieser Points wurden randomisiert, aber nach Altersjahrgang stratifiziert, Personen aus den Registern der Einwohnermeldeämter gezogen. Auf diese Weise wurden zunächst 150 Sample Points ermittelt, die bereits ein repräsentatives Abbild ermöglichen sollten. Später wurden 17 weitere Sample Points gezogen, um die Gesamtzahl der Teilnehmer aufzustocken. Für EsKiMo wurden jedoch nur die ursprünglichen 150 Sample Points berücksichtigt. Neben der deutschen Bevölkerung wurden auch Kinder und Jugendliche mit Migrationshintergrund eingeschlossen. Für eine ausführlichere Erläuterung des Konzeptes, Designs und der Methodik der KiGGS-Studie sei auf andere Publikationen verwiesen $[11,12,13,14]$.

Die an KiGGS anschließende Ernährungsstudie EsKiMo wurde im Jahr 2006 vom Robert Koch-Institut (RKI) und der Universität Paderborn mit finanzieller 


\begin{tabular}{|c|c|c|c|c|c|c|c|}
\hline & $\mathrm{N}^{*}$ & $\begin{array}{l}\text { Mittel- } \\
\text { wert }\end{array}$ & St. Abw. & Median & $P_{25}-P_{75}$ & $\begin{array}{l}\% \text { mit Konsum } \\
12-24 \mathrm{~g} / \mathrm{Tag}\end{array}$ & $\begin{array}{l}\% \text { mit Konsum } \\
>24 \mathrm{~g} / \mathrm{Tag}\end{array}$ \\
\hline \multicolumn{8}{|l|}{ Jungen } \\
\hline 12-13 J. & 219 & 0,1 & 0,4 & 0,0 & $0,0-0,0$ & $0 \%$ & $0 \%$ \\
\hline 14-15 J. & 209 & 2,2 & 7,1 & 0,0 & $0,0-0,9$ & $2,6 \%$ & $2,2 \%$ \\
\hline 16-17 J. & 194 & 10,4 & 17,8 & 4,9 & $0,9-12,4$ & $15,2 \%$ & $11,1 \%$ \\
\hline \multicolumn{8}{|l|}{ Mädchen } \\
\hline 12-13 J. & 215 & 0,1 & 0,2 & 0,0 & $0,0-0,0$ & $0 \%$ & $0 \%$ \\
\hline 14-15 J. & 234 & 1,6 & 4,7 & 0,0 & $0,0-0,6$ & $2,9 \%$ & $0,6 \%$ \\
\hline 16-17 J. & 201 & 3,8 & 7,2 & 1,3 & $0,0-5,9$ & $5,5 \%$ & $0,7 \%$ \\
\hline
\end{tabular}

Unterstützung des Bundesministeriums für Ernährung, Landwirtschaft und Verbraucherschutz umgesetzt. Es wurde eine Unterstichprobe der 6- bis 17-jährigen Kinder und Jugendlichen, die bereits an KiGGS teilgenommen hatten, angeschrieben und um die Teilnahme an EsKiMo gebeten. Die Teilnehmer erhielten zunächst ein Einladungsschreiben, in dem darauf hingewiesen wurde, dass die Angaben streng vertraulich nach den Bestimmungen des Bundesdatenschutzgesetzes behandelt und nur in anonymisierter Form gespeichert und ausgewertet werden. Dies wurde auch noch einmal in einer beiliegenden Datenschutzerklärung erläutert, woraufhin die Kenntnisnahme mit einer Einverständniserklärung bestätigt werden konnte. Die Adressdaten wurden lediglich für eine kurze persönliche Auswertung zur Nährstoffaufnahme einmalig mit den Lebensmittelangaben zusammengefügt und den Jugendlichen persönlich zugeschickt. Insgesamt nahmen 1234 Kinder im Alter von 6 bis 11 Jahren und 1272 Jugendliche im Alter von 12 bis 17 Jahren an der Studie teil.

Bei den 12 bis 17 Jahre alten Jugendlichen wurde eine detaillierte Erhebung über die Aufnahme von Lebensmitteln und Getränken, einschließlich alkoholischer Getränke, durchgeführt [15]. Während die Ergebnisse zu den 6- bis 11-jährigen Kindern aus den elterlichen Angaben in einem 3-Tage-Ernährungsprotokoll stammen, wurde die Ernährung der 12- bis 17-Jährigen mittels eines computergestützten „Face-to-Face“-Ernährungsinterviews, dem DISHES (Dietary Interview Software for Health Examination Studies), erfasst [16]. Diese Methode folgt dem Ta- gesablauf der Mahlzeiten und anderer Verzehrsgelegenheiten und hält dabei die Häufigkeiten und Portionsmengen der einzelnen Lebensmittel fest. Die Angaben und Getränke- beziehungsweise Alkoholkonsum beziehen sich auf die letzten vier Wochen vor den Interviews [15]. Bei den Auswertungen wurden alkoholische Getränke nach drei Kategorien differenziert: 1. Bier und Biermischgetränke, 2. Wein, weinhaltige Getränke und Sekt, 3. Spirituosen wie Likör beziehungsweise Likörwein, Branntwein, Cocktails und Alkopops. Aus Gründen der besseren Lesbarkeit werden die Kategorien im Folgenden als Bier, Wein und Spirituosen bezeichnet.

Des Weiteren wurden aus den detaillierten Angaben zu den konsumierten Getränken mithilfe des Bundeslebensmittelschlüssels Version 2.3 (BLS) die zugeführten Grammmengen an Alkohol pro Tag errechnet [17]. In dieser Lebensmittelinhaltstoffdatenbank sind Nährstoffdaten von über 10.000 einzelnen Lebensmitteln enthalten. Dazu zählen auch alkoholische Getränke, zum Beispiel Hefeweizenbier, Exportbier, Starkbier, trockener Weißwein, Rotwein oder Cognac. Diese Angaben sind genauso detailliert, wie die Angaben, die im DISHES-Interview erfragt wurden. Um die durchschnittliche Tagesmenge an reinem Alkohol zu berechnen, wurde der Alkoholgehalt gemäß folgender Formel mit den Mengenangaben und Häufigkeitsangaben multipliziert:

Alkohol pro Getränkeart (g pro $\mathrm{Tag}$ ) = Häufigkeit in 4 Wochen (Anzahl pro 28 Tage) $\times$ durchschnittliche Menge $(\mathrm{ml}) \times$ Alkoholgehalt $(\mathrm{g}$ pro $\mathrm{ml}) / 28$. der 12- bis 17-Jährigen zum Lebensmittel-
Danach wurden die Alkoholmengen pro Teilnehmer und pro Getränkegruppe summiert.

Zur Beschreibung von Gruppenunterschieden wurden die Merkmale Alter, Geschlecht, alte und neue Bundesländer, Sozialstatus, Schultyp und Migrationshintergrund betrachtet. Beim Wohnort wurde zwischen den alten und neuen Bundesländern (einschließlich Berlin) differenziert. Für die Zuordnung war ausschlaggebend, wo die Jugendlichen zum Zeitpunkt der Befragung lebten [18]. Zur Abschätzung des Sozialstatus wurde ein Index gebildet, der auf Angaben der Eltern über ihre schulische und berufliche Ausbildung, ihre berufliche Stellung sowie über das Haushaltsnettoeinkommen beruht. Diese drei Dimensionen wurden mit jeweils 1 bis 7 Punkten bewertet, sodass der als Punktsummenscore berechnete Index einen Wert zwischen 3 und 21 Punkten annehmen kann. Teilnehmern mit 3 bis 8 Punkten wurde ein niedriger Sozialstatus, mit 9 bis 14 Punkten ein mittlerer und mit 15 bis 21 Punkten ein hoher Sozialstatus zugewiesen. Demnach sind etwa die Hälfte der Kinder und Jugendlichen in Deutschland der mittleren Sozialstatusgruppe zuzurechnen, während auf die niedrige und hohe Sozialstatusgruppe ungefähr jeweils ein Viertel der Heranwachsenden entfällt [18]. Basierend auf den Informationen zum besuchten Schultyp beziehungsweise zum jeweils höchsten Schulabschluss, wurden die Jugendlichen nach Haupt-, Real-, Gesamtschüler oder Gymnasiasten eingeteilt [19]. Von einem Migrationshintergrund wurde ausgegangen, wenn die Jugendlichen selbst aus einem anderen Land zugewandert sind und mindestens ein Elternteil nicht in Deutschland geboren wurde oder wenn beide Eltern zugewandert beziehungsweise nichtdeutscher Staatsangehörigkeit sind. Nach dieser Definition weisen rund $17 \%$ der Kinder und Jugendlichen in Deutschland einen Migrationshintergrund auf [18].

Die statistische Auswertung erfolgte mit dem Statistikprogramm SAS Version 9.2. Mittelwerte und Standardabweichungen sowie Mediane, 25. und 75. Perzentile wurden für die durchschnittliche Tagesmenge an alkoholischen Getränken (Milliliter) und die Grammmenge an reinem Alkohol berechnet. Die 95\%-Kon- 
fidenzintervalle wurden mit der Surveyprozedur Proc Surveymeans berechnet, Regressionsmodelle sowie Student's t- und F-Tests mit Proc Surveyreg. Diese Surveyprozeduren können das komplexe Stichprobendesign, in diesem Fall die Clusterung nach Sample Points, berücksichtigen. In einfachen Regressionsmodellen wurde zunächst der Zusammenhang zwischen Alter, Geschlecht, neue und alte Bundesländer, Sozialstatus, Schultyp sowie Migrationshintergrund und dem Alkoholkonsum analysiert. Anschließend wurde in einem multiplen Regressionsmodell die unabhängige Beziehung dieser Variablen mit dem Alkoholkonsum untersucht. Für diese Regression wurde eine Wurzeltransformation (die 3. Wurzel) des Gesamtalkoholkonsums vorgenommen, die dann als abhängige Variable eingesetzt wurde. Die so transformierten Werte sind annähernd normal verteilt. Von einem signifikanten Effekt wurde ausgegangen, wenn der t-Test beziehungsweise F-Test ein Signifikanzniveau von $\mathrm{p}<0,05$ auswies (Werte zwischen 0,10 und 0,05 wurden als grenzwertig signifikant eingestuft). Kategorielle Variablen wurden als sogenannte „Class"-Variablen in das Modell aufgenommen, mit Mädchen, alte Bundesländer, niedriger Sozialstatus, Nichtmigrant und Gymnasium als jeweilige Referenz (Bezugsgruppe). Dies entspricht zum Beispiel für den Sozialstatus die Bildung von jeweils einem Dummy für mittleren und hohen Sozialstatus und für den Schultyp drei Dummys für Haupt-, Real- und Gesamtschule.

Zur Gewährleistung der Repräsentativität wurden die Analysen mit einem Gewichtungsfaktor durchgeführt. Dieser Faktor gewichtet jeden Teilnehmer, um Abweichungen der Nettostichprobe von der Bevölkerungsstruktur (Stand: 31.12.2004) hinsichtlich Alter (in Jahren), Geschlecht, Region (Ost/West/Berlin) und Staatsangehörigkeit zu korrigieren [13].

\section{Ergebnisse}

In $\bullet$ Tab. 1 sind einige Kennzahlen des Alkoholkonsums Jugendlicher nach Alter und Geschlecht dargestellt. Diese weisen auf eine starke Zunahme der durchschnittlich am Tag aufgenommenen Alko-

Bundesgesundheitsbl 2009·52:745-752 DOI 10.1007/s00103-009-0876-7

(c) Springer-Verlag 2009

\section{S. Kohler · A. Richter · T. Lampert · G.B.M. Mensink Alkoholkonsum bei Jugendlichen in Deutschland. Ergebnisse aus EsKiMo}

\section{Zusammenfassung}

Die Studie EsKiMo (Ernährungsstudie als KiGGS-Modul) wurde in der Zeit von Januar bis Dezember 2006 als Nachfolgeerhebung des Kinder- und Jugendgesundheitssurveys (KiGGS) durchgeführt. Mit Daten aus dieser Studie wird der Alkoholkonsum von Jugendlichen in Deutschland untersucht. Insgesamt wurden 1272 Jungen und Mädchen im Alter von 12 bis 17 Jahren mithilfe des Ernährungserhebungsprogramms DISHES, das auf der "diet history methode" basiert, befragt. Aus dem Konsum der letzten vier Wochen wurden Mittelwerte und Perzentile der durchschnittlichen Tagesmengen an alkoholischen Getränken und reinem Alkohol, differenziert nach Alter, Geschlecht, neuen und alten Bundesländern, Sozialstatus, Schultyp und Migrationshintergrund, berechnet. Der Zusammenhang zwischen diesen Variablen und dem Alkoholkonsum wurde in univariaten Analysen und in einer multiplen linearen Regression ermittelt. Von den alkoholischen $\mathrm{Ge}$ tränken wird mengenmäßig am häufigsten Bier getrunken. Bei den 16- bis 17-Jäh- rigen nehmen Jungen im Mittel 10,4 g Alkohol, Mädchen 3,8 g Alkohol pro Tag auf, wobei $11 \%$ der Jungen und $6 \%$ der Mädchen sogar die Grenzwerte für riskante Trinkmengen, die für Erwachsene gelten, überschreiten. Der Alkoholkonsum steigt signifikant mit dem Alter $(p<0,001)$, Jungen trinken signifikant $(p<0,001)$ mehr Alkohol als gleichaltrige Mädchen und Migranten signifikant weniger als Nicht-Migranten $(p<0,05)$. Die Unterschiede zwischen den neuen und alten Bundesländern sowie nach Sozialstatus und Schultyp sind statistisch nicht signifikant. Der Alkoholkonsum unter Jugendlichen ist hoch, besonders unter 16- bis 17-jährigen Jungen. Da etwa ein Viertel der 12- bis 13-Jährigen mindestens einmal in den letzten vier Wochen Alkohol getrunken hat, gibt es bereits bei dieser Gruppe Handlungsbedarf.

\section{Schlüsselwörter}

EsKiMo · Jugendliche · Alkohol · Deutschland · Survey

\section{Alcohol consumption among adolescents in Germany. Results of EsKiMo}

Abstract

The study EsKiMo (Eating study as a KiGGS

Module) was conducted from January to December 2006 as a follow-up study of the German Health Interview and Examination Survey for Children and Adolescents (KiGGS). With data from this study, the alcohol consumption of adolescents in Germany is explored. Interviews were conducted with 1272 adolescents, aged 12 to 17 years, using the dietary assessment software DISHES which is based on the diet history method. From consumption data of the previous four weeks, means and percentiles of the average daily consumption of alcoholic beverages as well as pure alcohol intake were calculated, stratified by age, sex, new and old federal states, social status, type of school, and migration background. Furthermore, the associations of these variables with alcohol intake were determined in univariate and multiple linear regression analyses. The highest amount of alcoholic beverages is consumed as beer.
Among 16- to 17-year-olds, boys consume on average $10.4 \mathrm{~g}$ alcohol and girls $3.8 \mathrm{~g}$ alcohol per day and even $11 \%$ of boys and $6 \%$ of girls exceed the tolerable upper intake levels for adults. Alcohol consumption increases significantly with age $(p<0.001)$, boys drink significantly more alcohol than girls of the same age $(p<0.001)$, and migrants significantly less than non-migrants $(p<0.05)$. Differences between new and old federal states, social status, and type of school are not statistically significant. Alcohol consumption is high among adolescents, most particular among boys aged 16 to 17 years. Since about a quarter of 12- to 13-year-olds consumed alcohol at least once in the last four weeks, there is a need for action already in this age group.

Keywords

EsKiMo · Adolescents · Alcohol · Germany · Survey 
Tab. 2 Durchschnittlicher Konsum alkoholischer Getränke in ml pro Tag bei 12- bis 17-jährigen Konsumenten, Anteil Konsumenten (\%), Mittelwerte (MW), Mediane und Interquartilbereiche, Konsum innerhalb der letzten vier Wochen

\begin{tabular}{|c|c|c|c|c|c|c|c|c|c|c|c|c|c|c|c|c|}
\hline & \multicolumn{3}{|c|}{ Alle Getränke } & \multicolumn{4}{|c|}{ Bier } & \multicolumn{3}{|c|}{ Wein } & \multicolumn{6}{|c|}{ Spirituosen } \\
\hline & $\%$ & MW & $\begin{array}{l}\text { Medi- } \\
\text { an }\end{array}$ & $P_{25}-P_{75}$ & $\%$ & MW & $\begin{array}{l}\text { Medi- } \\
\text { an }\end{array}$ & $P_{25}-P_{75}$ & $\%$ & MW & $\begin{array}{l}\text { Medi- } \\
\text { an }\end{array}$ & $P_{25}-P_{75}$ & $\%$ & MW & $\begin{array}{l}\text { Medi- } \\
\text { an }\end{array}$ & $P_{25}-P_{75}$ \\
\hline \multicolumn{17}{|l|}{ Jungen } \\
\hline $12-13 \mathrm{~J}$. & 23,1 & 8,0 & 0,3 & $0,1-2,9$ & 4,14 & 37,2 & 21,4 & $11,8-35,7$ & 6,6 & 2,6 & 0,6 & $0,1-2,9$ & 15,6 & 0,9 & 0,2 & $0,1-0,4$ \\
\hline $14-15 \mathrm{~J}$. & 51,1 & 118,3 & 23,6 & $1,4-160,7$ & 33,0 & 167,1 & 71,4 & $23,6-214,3$ & 13,7 & 11,1 & 3,0 & $1,4-13,5$ & 27,0 & 13,9 & 1,1 & $0,1-10,7$ \\
\hline 16-17 J. & 84,3 & 273,0 & 188,6 & $71,4-344,6$ & 77,6 & 254,7 & 178,6 & $85,7-321,4$ & 23,9 & 24,7 & 13,4 & $5,7-26,8$ & 52,5 & 50,7 & 17,9 & $2,9-57,1$ \\
\hline \multicolumn{17}{|l|}{ Mädchen } \\
\hline $12-13 \mathrm{~J}$. & 22,9 & 3,1 & 0,3 & $0,1-2,3$ & 3,6 & 10,3 & 6,4 & $0,7-17,9$ & 8,6 & 3,3 & 1,4 & $0,4-2,9$ & 14,6 & 0,5 & 0,1 & $0,1-0,1$ \\
\hline 14-15 J. & 53,7 & 66,5 & 12,3 & $0,8-53,6$ & 26,8 & 98,5 & 47,1 & $11,8-107,1$ & 20,0 & 17,9 & 5,4 & $2,9-17,9$ & 34,3 & 16,7 & 0,4 & $0,1-4,5$ \\
\hline 16-17 J. & 73,8 & 97,2 & 42,1 & $17,1-153,9$ & 47,8 & 93,4 & 47,5 & $23,6-142,9$ & 39,1 & 20,3 & 10,0 & $4,3-26,8$ & 51,0 & 37,8 & 12,1 & $2,9-43,1$ \\
\hline
\end{tabular}

Tab. 3 Alkoholkonsum in g pro Tag nach Wohnregion, Sozialstatus, Migrationshintergrund und Schultyp bei 14- bis 17-Jährigen, Mittelwerte (MW) und 95\%-Konfidenzintervalle (KI), Konsum innerhallb der letzten 4 Wochen

\begin{tabular}{|c|c|c|c|c|c|c|c|c|c|c|c|c|}
\hline & \multicolumn{6}{|c|}{ Jungen } & \multicolumn{6}{|c|}{ Mädchen } \\
\hline & \multicolumn{3}{|c|}{ 14-15 Jahre } & \multicolumn{3}{|c|}{ 16-17 Jahre } & \multicolumn{3}{|c|}{ 14-15 Jahre } & \multicolumn{3}{|c|}{ 16-17 Jahre } \\
\hline & $\mathrm{n}^{\mathrm{a}}$ & MW (KI) & $p^{b}$ & $\mathrm{n}^{\mathrm{a}}$ & MW (KI) & $\mathbf{p}^{\mathbf{b}}$ & $n^{a}$ & MW (KI) & $p^{b}$ & $\mathrm{n}^{\mathrm{a}}$ & MW (KI) & $p^{b}$ \\
\hline \multicolumn{13}{|l|}{ Wohnregion } \\
\hline Neue Bundesländer & 72 & $2,6(0,2-5,1)$ & ns & 73 & $10,5(5,9-15,0)$ & ns & 74 & $2,1(0,6-3,5)$ & ns & 67 & $3,0(1,8-4,3)$ & ns \\
\hline Alte Bundesländer & 137 & $2,0(1,1-3,0)$ & & 121 & $10,4(7,6-13,3)$ & & 160 & $1,5(0,7-2,3)$ & & 134 & $4,0(2,8-5,1)$ & \\
\hline \multicolumn{13}{|l|}{ Sozialstatus } \\
\hline Niedrig & 43 & $1,1(0,1-2,2)$ & ns & 44 & $12,5(4,7-20,4)$ & ns & 55 & $0,9(0,2-1,5)$ & ns & 40 & $2,0(0,9-3,1)$ & 0,01 \\
\hline Mittel & 105 & $2,2(1,1-3,4)$ & & 98 & $9,1(6,7-11,6)$ & & 130 & $2,2(0,9-3,4)$ & & 94 & $4,5(3,1-5,9)$ & \\
\hline Hoch & 58 & $3,2(0,6-5,8)$ & & 49 & $11,7(7,3-16,1)$ & & 47 & $1,1(0,3-1,8)$ & & 64 & $4,0(2,0-6,0)$ & \\
\hline \multicolumn{13}{|l|}{ Schultyp } \\
\hline Hauptschule & 39 & $1,6(0,4-2,8)$ & 0,06 & 43 & $12,2(4,9-19,5)$ & ns & 28 & $0,8(0,1-1,5)$ & ns & 23 & $4,7(2,2-7,2)$ & 0,01 \\
\hline Realschule & 72 & $3,0(1,0-5,0)$ & & 69 & $11,1(8,0-14,2)$ & & 80 & $1,4(0,4-2,5)$ & & 63 & $3,1(1,6-4,5)$ & \\
\hline Gymnasium & 69 & $2,5(0,6-4,4)$ & & 62 & $7,9(4,2-11,6)$ & & 97 & $2,2(0,7-3,6)$ & & 94 & $4,5(2,9-6,1)$ & \\
\hline Gesamtschule & 23 & $0,7(0,1-1,4)$ & & 11 & $11,1(-2,6-4,9)$ & & 25 & $1,3(-0,2-2,6)$ & & 13 & $1,2(-0,4-2,8)$ & \\
\hline \multicolumn{13}{|c|}{ Migrationshintergrund } \\
\hline $\mathrm{Ja}$ & 18 & $0,7(-0,7-2,1)$ & 0,05 & 18 & $8,4(2,0-14,8)$ & ns & 31 & $1,9(-1,4-5,1)$ & ns & 24 & $2,3(0,8-3,8)$ & 0,03 \\
\hline Nein & 191 & $2,4(1,4-3,4)$ & & 176 & $10,7(8,1-13,3)$ & & 203 & $1,5(1,0-2,1)$ & & 177 & $4,0(3,0-5,1)$ & \\
\hline
\end{tabular}

holmenge mit dem Alter hin. Für die 16bis 17-jährigen Jungen ergab sich ein Mittelwert von 10,4 g pro Tag (Median 4,9) und bei den gleichaltrigen Mädchen von 3,8 g pro Tag (Median 1,3). Allein 11,1\% der 16- bis 17-jährigen Jungen nahmen mehr als $24 \mathrm{~g}$ Alkohol pro Tag auf (oberer tolerabler Grenzwert für Männer) [20, 21], bei den Mädchen entsprechenden Alters konsumierten 6,2\% mehr als $12 \mathrm{~g}$ Alkohol pro Tag (oberer tolerabler Grenzwert für Frauen) [20, 21].

$54 \%$ der 12- bis 17-jährigen Jungen und $51 \%$ der gleichaltrigen Mädchen gaben für die letzten vier Wochen vor den Interviews an, alkoholische Getränke getrunken zu haben. Bei den 12- bis 13-jährigen
Jungen und Mädchen war dieser Anteil mit jeweils $23 \%$ deutlich geringer als bei den 14- bis 15-Jährigen (Jungen 51\%, Mädchen $54 \%$ ) und insbesondere als bei den 16- bis 17-Jährigen (Jungen 84\%, Mädchen $74 \%)$.

In - Tab. 2 werden Prozentanteile der Konsumenten sowie Mediane, 25. und 75. Perzentile der durchschnittlichen Tagesaufnahmen der jeweiligen Getränkesorten dargestellt. Dabei zeigten sich deutliche Geschlechterunterschiede. So gaben $48 \%$ der 16- bis 17-jährigen Mädchen an, Bier zu trinken, im Vergleich zu $78 \%$ der Jungen gleichen Alters. Der Anteil an Weinkonsumenten war in dieser Altersgruppe bei Mädchen mit 39\% höher als bei Jungen mit $24 \%$. Die Werte zu den Spirituosen waren bei den 16- bis 17-jährigen Mädchen (51\%) und Jungen (53\%) fast gleich hoch.

Von den Jugendlichen die alkoholische Getränke konsumierten, tranken in der Altersgruppe der 14- bis 15-Jährigen Jungen im Mittel 118,3 $\mathrm{ml}$ pro Tag (Median 23,6) und Mädchen $66,5 \mathrm{ml}$ pro Tag (Median 12,3). In der Gruppe der 16- bis 17Jährigen lagen diese Werte mit 273,o ml pro Tag (Median 188,6) bei Jungen und 97,2 ml pro Tag (Median 42,1) bei Mädchen deutlich höher. In Bezug auf die einzelnen Getränkesorten zeigten sich die größten geschlechtsspezifischen Unterschiede beim Bierkonsum der 16- bis 17- 
Jährigen. Jungen dieses Alters tranken im Mittel 254,7 ml Bier pro Tag (Median 178,6), der Vergleichswert für Mädchen lag bei 93,4 $\mathrm{ml}$ pro Tag (Median 47,5).

Die - Abb. 1 zeigt den durchschnittlichen Beitrag der einzelnen Getränkekategorien zum gesamten Alkoholkonsum. Aufgrund der vergleichsweise niedrigen Mengen, die von den 12- bis 13-Jährigen konsumiert werden, sind nur die Ergebnisse für die 14- bis 17-Jährigen dargestellt. Bei den Jungen wurde der Alkohol meist über Bier, bei den 16- bis 17-jährigen Mädchen vor allem über Spirituosen aufgenommen.

\section{Univariate Betrachtung}

In - Tab. 3 wird der mittlere Alkoholkonsum, differenziert nach möglichen Determinanten, dargestellt. Bei Mädchen im Alter von 14 bis 15 Jahren war der diesbezügliche Mittelwert in den neuen Bundesländern mit 2,1 g pro Tag etwas höher als in den alten Bundesländern mit 1,5 g pro Tag. Umgekehrt war der Mittelwert bei den 16- bis 17-jährigen Mädchen in den alten Bundesländern mit $4 \mathrm{~g}$ pro Tag höher als in den neuen Bundesländern mit $3 \mathrm{~g}$ pro Tag. Der Alkoholkonsum in Gramm pro Tag unterscheidet sich jedoch nicht signifikant zwischen den neuen und alten Bundesländern.

Die 14 bis 15 Jahre alten Jugendlichen mit niedrigem Sozialstatus tranken im Vergleich zu den Gruppen mit mittlerem beziehungsweise hohem Sozialstatus tendenziell (nicht signifikant) weniger Alkohol. Bei den 16 bis 17 Jahre alten Jungen war kein Trend hinsichtlich Sozialstatus erkennbar, bei gleichaltrigen Mädchen tranken jedoch diejenigen mit niedrigem Sozialstatus signifikant weniger Alkohol. Bei den 14- bis 15-Jährigen nahmen sowohl Jungen als auch Mädchen, die die Hauptschule oder Gesamtschule besuchten, am wenigsten Alkohol und die Gymnasiasten am meisten Alkohol zu sich. Bei Jungen waren diese Unterschiede grenzwertig signifikant. Die Fallzahl an Schülern der Gesamtschule ist relativ gering, weshalb die Ergebnisse bei dieser Gruppe mit Vorsicht interpretiert werden sollten. In der Altersgruppe der 16- bis 17-Jährigen konsumierten die Hauptschüler die höchsten Alkoholmengen (Jungen 12,2 g pro

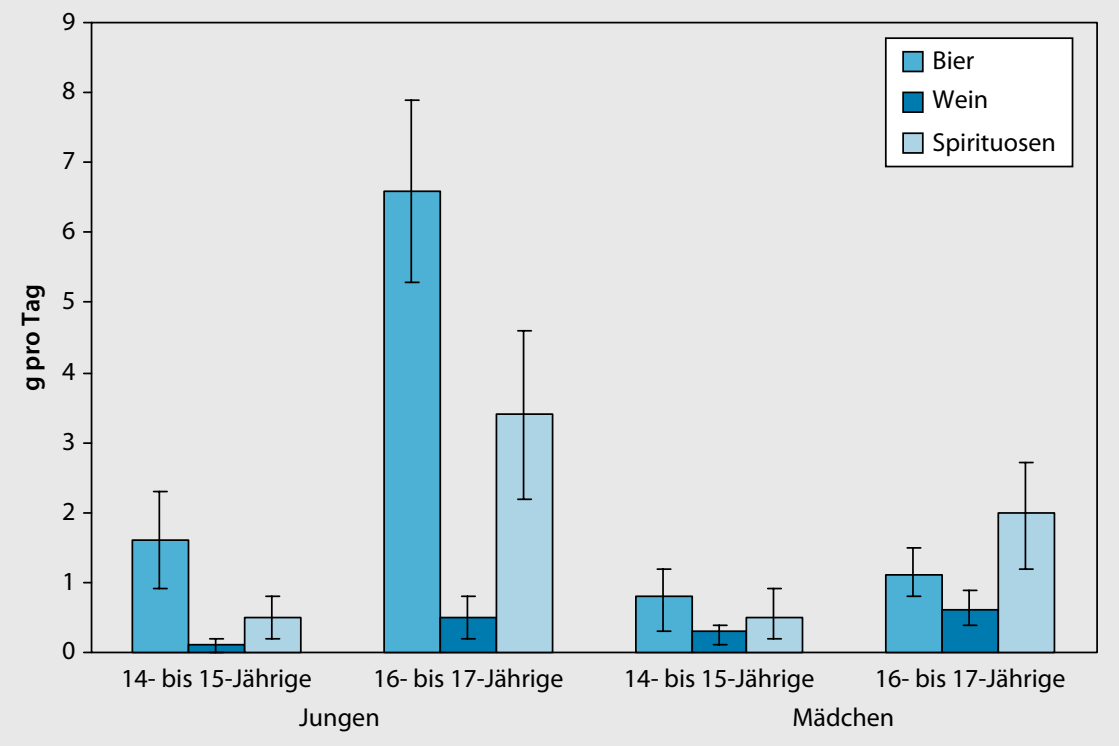

Abb. $1 \Delta$ Alkoholkonsum in g pro Tag nach Getränkeart bei 14- bis 17-Jährigen, Mittelwerte und 95\%Konfidenzintervalle, Konsum innerhalb der letzten vier Wochen

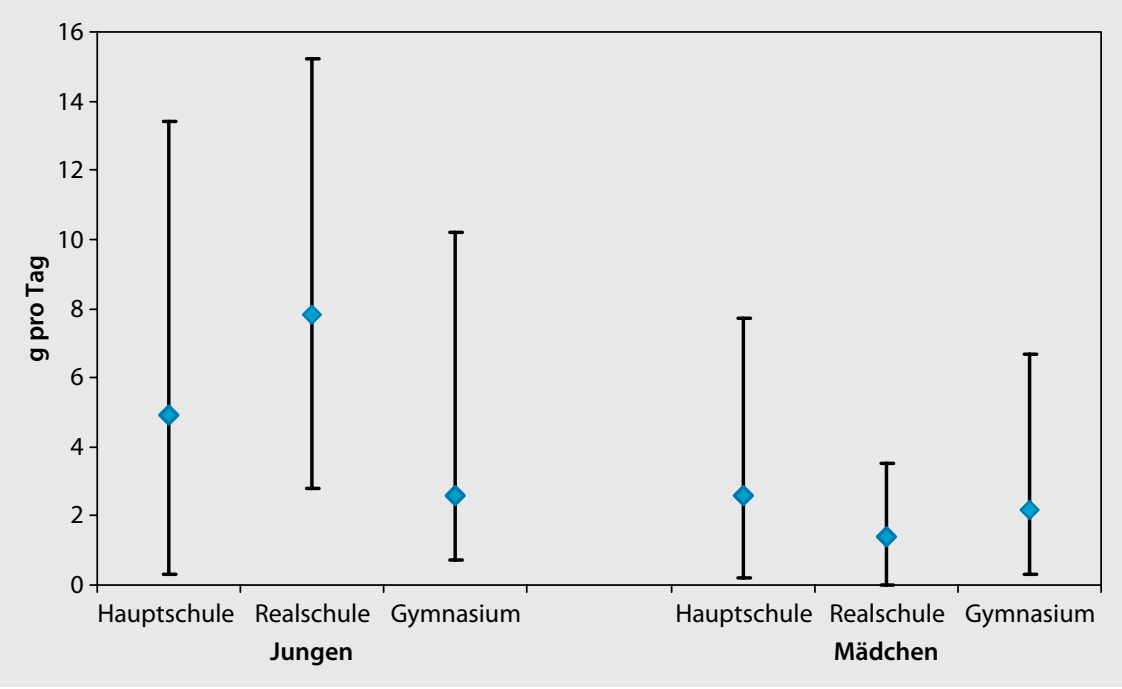

Abb. $2 \Delta$ Alkoholkonsum in g pro Tag nach Schultyp bei 16- bis 17-Jährigen, Mediane und 25. bis 75. Perzentilbereich, Konsum innerhalb der letzten vier Wochen

Tag, Mädchen 4,7 g pro Tag). Beim Vergleich der Mediane zeigt sich allerdings die höchste Aufnahmemenge bei 16- bis 17-jährigen Realschülern (• Abb. 2). Bei den weiblichen Jugendlichen waren die Differenzen nach Schultyp signifikant, was an der geringeren Alkoholaufnahme in der Gruppe der Gesamtschüler lag. Abgesehen von den 14- bis 15-jährigen Mädchen, konsumierten Jugendliche mit Migrationshintergrund im Vergleich zu den Jugendlichen ohne Migrationshintergrund weniger alkoholische Getränke. Bei 14- bis 15-jährigen Jungen und bei 16bis 17-jährigen Mädchen war diese Diffe- renz statistisch signifikant. Bei den 16- bis 17-jährigen Mädchen zeigten sich signifikante Differenzen im Alkoholkonsum für Sozialstatus, Migrationshintergrund und Schultyp.

\section{Multivariate Betrachtung}

Mittels eines linearen Regressionsmodells wurden die Determinanten gemeinsam betrachtet (- Tab. 4). Weil die transformierten Regressionskoeffizienten nicht direkt quantitativ interpretierbar sind, werden diese hier nicht dargestellt. Insgesamt geht aus den durchgeführten Analysen hervor, 
Tab. 4 Ergebnisse der linearen Regression mit transformiertem Alkoholkonsum (3. Wurzel der Grammmengen) als abhängige Variable, Konsum innerhalb der letzten 4 Wochen, Alter 14-17 Jahre

\begin{tabular}{|c|c|c|}
\hline Parameter & $t$-Wert & p-Wert \\
\hline Intercept & $-11,53$ & $<, 0001$ \\
\hline Alter in Jahren & 12,83 & $<, 0001$ \\
\hline Jungen $^{a}$ & 4,36 & $<, 0001$ \\
\hline Neue Bundesländer & $-0,59$ & 0,5548 \\
\hline Hoher Sozialstatus & 1,52 & 0,1319 \\
\hline Mittlerer Sozialstatus & 1,73 & 0,0849 \\
\hline Hauptschule & 1,33 & 0,1865 \\
\hline Realschule & 1,69 & 0,0922 \\
\hline Gesamtschule & $-0,80$ & 0,4236 \\
\hline Migrant & $-2,00$ & 0,0475 \\
\hline \multicolumn{3}{|c|}{$\begin{array}{l}\mathrm{R}^{2}=0,25,9 \text { Freiheitsgrade im Model; a Referenz sind } \\
\text { Mädchen, alte Bundesländer, niedriger Sozialstatus } \\
\text { Nichtmigrant und Gymnasium }\end{array}$} \\
\hline
\end{tabular}

dass mit zunehmendem Alter signifikant mehr Alkohol aufgenommen wurde und Jungen signifikant mehr Alkohol tranken als Mädchen bei Konstanthaltung aller im Modell aufgenommenen Variablen. Wohnregion und Schultyp zeigten keinen signifikanten Zusammenhang mit der Alkoholaufnahme. Die Jugendlichen mit einem hohen und mittleren Sozialstatus nahmen tendenziell (nicht signifikant) mehr Alkohol auf als die mit niedrigem. Bei Konstanthaltung von Alter, Geschlecht, Sozialstatus und Schultyp konsumierten Jugendliche mit Migrationshintergrund signifikant weniger Alkohol als Jugendliche ohne Migrationshintergrund.

\section{Diskussion}

Die Ergebnisse zeigen, dass mehr als die Hälfte der 12- bis 17-jährigen Jugendlichen in den vier Wochen vor der Befragung mindestens einmal Alkohol getrunken hatte. Bei den 12- bis 13-Jährigen lag dieser Anteil bereits bei $23 \%$. Unter den 16 bis 17 -Jährigen trinken $11 \%$ der Jungen im Durchschnitt mehr als $24 \mathrm{~g}$ Alkohol pro Tag und 6\% der Mädchen mehr als $12 \mathrm{~g}$ Alkohol pro Tag. Diese Mengen gelten als obere gesundheitliche Unbedenklichkeitsgrenze für erwachsene Männer beziehungsweise Frauen [20, 21]. Für Jugendliche gilt jedoch die Empfehlung, Alkohol weitgehend zu meiden [21]. Somit ist der frühzeitige und hohe Alkoholkonsum der Jugendlichen ein bedeutendes Gesund- heitsproblem. Alkoholkonsum in diesem Lebensabschnitt kann zu erheblichen körperlichen Schäden führen. Außerdem ist das frühe Jugendalter eine Phase, in der Verhaltensweisen noch stark vom jeweiligen Umfeld beeinflusst werden - möglicherweise auch in einer unerwünschten Weise. Diese können zu pathologischen Alkoholkonsummustern führen, die mit den bekannten Folgeschäden einhergehen $[2,3]$.

Die starke Verbreitung des Alkoholkonsums bei Jugendlichen in Deutschland wird durch andere Studien bestätigt $[22,23$, $24,25,26]$. Diese erlauben zudem genaue Angaben zum Alter bei Erstkonsum. So zeigt die Drogenaffinitätsstudie der BZgA aus dem Jahr 2004, dass das Durchschnittsalter, in dem Jugendliche anfangen, Alkohol zu konsumieren, bei 14 Jahren liegt [26]. Europaweit liegt dieser Durchschnitt bei etwa 12,5 Jahren [27]. Untersuchungen in den USA weisen auf einen Zusammenhang zwischen frühem Einstiegsalter und der Prävalenz von Alkoholmissbrauch, Alkoholabhängigkeit sowie stressbedingtem Alkoholkonsum im späteren Leben hin [2, 3]. Ob hier tatsächlich eine Kausalität besteht, ist aber noch zu klären.

Die Ergebnisse von EsKiMo weisen auf nur geringe Unterschiede im Alkoholkonsum Jugendlicher nach Sozialstatus, besuchtem Schultyp und Migrationshintergrund hin. In der multivariaten Betrachtung zeigte sich als signifikantes Ergebnis lediglich, dass Jugendliche mit Migrationshintergrund weniger Alkohol konsumieren als Gleichaltrige ohne Migrationshintergrund. Hier ist die heterogene Zusammensetzung der Gruppe der Migranten zu beachten, das heißt, je nach Herkunftsland sowie kulturellem und religiösem Hintergrund kommt dem Alkohol als Genussmittel unterschiedliche Bedeutung zu. Vertiefende Analysen der in der KiGGS-Hauptstudie erhobenen Daten zeigten, dass vor allem Jugendliche mit islamischem Hintergrund im Vergleich zu deutschen Heranwachsenden einen geringeren Alkoholkonsum haben [28].

Innerhalb Europas wurde kein konsistenter Trend für sozialstatusspezifische Unterschiede im Alkoholkonsum Jugendlicher gefunden. Auch wurde beobachtet, dass der soziale Status wenig Bedeutung für die Prävalenz von Trunken- heit hat, diesbezügliche Differenzen fanden sich am ehesten noch bei Jungen [29]. Hingegen wurden deutlich häufiger problematische Trinkmuster bei Erwachsenen mit niedrigem Sozialstatus gefunden. Ein möglicher Grund für diese Diskrepanz könnte der geringere finanzielle Handlungsspielraum sozial benachteiligter Jugendlicher sein [27].

Die Ergebnisse deuten also darauf hin, dass Jugendliche generell als Risikogruppe zu betrachten sind, und zwar weitgehend unabhängig vom Sozialstatus und besuchten Schultyp. Besonders gefährdet für einen hohen Alkoholkonsum sind 16bis 17-jährige Jungen. Zu den Gruppen mit einem relativ geringen Risiko zählen Jugendliche mit islamischem Hintergrund.

Bei der Interpretation der Ergebnisse sind außerdem einige methodische Aspekte zu berücksichtigen. So muss bei Befragungen zum Alkoholkonsum immer mit nicht wahrheitsgemäßen Angaben gerechnet werden. Dies ist teilweise auf Erinnerungslücken, die Unregelmäßigkeit des Konsums und die Befragungsmethode zurückzuführen. Da ein hoher Alkoholkonsum ein sozial nicht wünschenswertes Verhalten darstellt, werden die tatsächlich getrunkenen Mengen häufig unterschätzt (Underreporting). Im Gegensatz dazu werden aber wahrscheinlich von einem Teil der Jugendlichen auch übertriebene Angaben zum Alkoholkonsum gemacht, da sie einen solchen als vermeintliche Stärke betrachten. Da die Teilnehmer ausdrücklich darüber informiert wurden, dass die Befragung anonym ist, und die Abfrage des Alkoholkonsums im Rahmen einer ausführlichen Ernährungsbefragung erfolgte, gehen wir jedoch davon aus, dass uns relativ wahrheitsgemäße Angaben vorliegen. Eine Stärke der Studie ist, dass die alkoholischen Getränke quantitativ erfasst und nach verschiedenen Getränkekategorien eingeteilt wurden. Außerdem können durch die Verknüpfung mit KiGGS die Zusammenhänge zwischen Alkoholkonsum und einer Vielzahl an Determinanten untersucht werden.

Die Ergebnisse machen erneut deutlich, dass umfangreiche Präventionsmaßnahmen zum risikoreichen Umgang Jugendlicher mit Alkohol genauso notwendig sind wie Strategien gegen den Alkoholkonsum bei Kindern. Wichtig ist, 
dass die Maßnahmen bereits früh ansetzen und Kinder und Jugendliche in ihren zentralen Lebenswelten erreichen, also zum Beispiel in der Familie, in der Schule und in Sportvereinen. Nach wissenschaftlichen Erkenntnissen ist ein multipler Ansatz aus unterschiedlichen, aber aufeinander abgestimmten Maßnahmen am aussichtsreichsten [30]. Ebenso ist die Verknüpfung struktureller und personenbezogener präventiver Maßnahmen sinnvoll $[31,32]$.

Breit gestreute (massenmediale) Aufklärungskampagnen können das Bewusstsein über die negativen Folgen des Alkoholkonsums erhöhen. Die bisherigen Erfahrungen zeigen aber, dass ihre konkreten Auswirkungen auf das Konsumverhalten häufig gering sind [27]. Deshalb erscheint eine weitere Diskussion über ihre Inhalte, Zielgruppen und Zugangswege angezeigt, wobei Forschungsergebnisse sehr hilfreich sein können. Auch kommt den strukturellen Rahmenbedingungen eine erhebliche Bedeutung zu. Möglichkeiten ergeben sich hier zum Beispiel über straßenverkehrsbezogene Regelungen (wie niedrige Promillegrenzen für jugendliche Fahrer, häufige Blutalkoholtests), preisregulierende Maßnahmen (über Alkoholsteuer) und Maßnahmen, die Jugendlichen den Zugang zu alkoholischen Getränken erschweren. Im Interesse der Kinder- und Jugendgesundheit wird daher eine strikte Einhaltung des gesetzlichen Mindestalters und eine Einschränkung der Verkaufszeiten für alkoholische Getränke empfohlen (zum Beispiel in Bars, Restaurants, Supermärkten, Tankstellen) [27]. Gleichzeitig ist es erforderlich, das Einhalten des Jugendschutzes verstärkt zu kontrollieren [32]. Da Jugendliche besonders sensibel auf Preiserhöhungen reagieren, sollten die Alkoholsteuern für alle alkoholischen Getränke (nicht nur für Alkopops) spürbar angehoben werden. Beschränkt man die Erhöhung lediglich auf einzelne Getränkearten, weichen Jugendliche auf andere Alkoholsorten aus [31]. Wichtig erscheint in diesem Zusammenhang auch die Erkenntnis, dass Alkoholwerbung das Trinkverhalten von Kindern und Jugendlichen beeinflusst $[33,34,35]$ und Werbeverbote $\mathrm{zu}$ einer Reduzierung des Alkoholkonsums beitragen können [30].
Erfreulicherweise haben die Bemühungen zur Alkoholprävention in den letzten Jahren zugenommen. Im Vergleich zu anderen Ländern besteht in Deutschland diesbezüglich aber immer noch Nachholbedarf $[30,31,32]$. Unabdingbare Voraussetzung für die Gestaltung von Präventionsmaßnahmen ist das Vorliegen von Daten, die zeitliche Veränderungen und Entwicklungen kenntlich machen. Mit regelmäßig bereitgestellten repräsentativen und aktuellen Studienergebnissen ist es möglich, effektiv und nachhaltig zu intervenieren und den Erfolg der umgesetzten Maßnahmen zu kontrollieren. Es ist daher wichtig, die in Deutschland vorhandenen Erhebungen fortzuführen und für die Alkohol- und Suchtprävention zu nutzen.

\section{Schlussfolgerungen}

Die vorliegende Studie zeigt, dass viele 12- bis 13-Jährige bereits Alkohol getrunken haben, etwa ein Viertel mindestens einmal innerhalb der letzten vier Wochen vor ihrer Befragung. Diese Anteile sowie die durchschnittlich getrunkenen Mengen steigen mit zunehmendem Alter rasant an. Von den 16- bis 17-Jährigen trinken $11 \%$ der Jungen und $6 \%$ der Mädchen durchschnittliche Mengen, die für Erwachsene als gesundheitlich bedenklich gelten. Migranten konsumieren generell weniger Alkohol als Nichtmigranten. Nennenswerte Unterschiede zwischen Sozialstatusgruppen, neuen und alten Bundesländern sowie nach besuchtem Schultyp gibt es jedoch nicht. Die Ergebnisse unterstreichen die Bedeutung spezifischer Maßnahmen zur Alkoholprävention im Jugendalter. Diese sollten außer auf eine grundsätzliche Verringerung des Alkoholkonsums insbesondere auch auf die Vermeidung eines frühen Einstiegs in den Konsum alkoholischer Getränke zielen.

\section{Korrespondenzadresse}

\section{G.B.M. Mensink}

Abteilung für Epidemiologie und Gesundheitsberichterstattung, Robert Koch-Institut

Postfach 650261

13302 Berlin

g.mensink@rki.de

Interessenkonflikt. Der korrespondierende Autor gibt an, dass kein Interessenkonflikt besteht.

\section{Literatur}

1. Settertobulte W (2003) Problematische Formen des Alkoholkonsums - Häufigkeiten, Trends, Ursachen. In: Farke W, Graß H, Hurrelmann K (Hrsg) Drogen bei Kindern und Jugendlichen. Legale und illegale Substanzen in der ärztlichen Praxis. Thieme, Stuttgart, S 81-85

2. Dawson A, Grant B, Li T (2007) Impact of age at first drinking on stress-reactive drinking alcoholism. Clin Exp Res 31(1):69-77

3. Grant B, Dawson D (1997) Age at onset of alcohol use and its association with DSM-IV alcohol abuse and dependence: results from the national longitudinal alcohol epidemiologic survey. J Subst Abus 9:103-110

4. Bode C, Bode JC (1999) Protektive Wirkung und Missbrauch von Alkohol. In: Biesalski HK et al (Hrsg) Ernährungsmedizin. 2. überarbeitete Aufl. Thieme, Stuttgart New York, S 516-538

5. Ravens-Sieberer U, Nickel J (2008) Drogenaffinitätsstudie, Repräsentativerhebung, HBSC, ESPAD, Kinder- und Jugendsurvey: Was wissen wir über jugendlichen Alkoholkonsum? In: Deutsche Hauptstelle für Suchtfragen (Hrsg) Voll drauf neue Formen jugendlichen Alkoholkonsums: Dokumentation zur Tagung der Drogenbeauftragten der Bundesregierung in Berlin 2007, S 14-25

6. Gmel G, Rehm J, Kuntsche E (2003) Binge drinking in Europe: definitions, epidemiology and consequences. SUCHT 49(2):105-116

7. Bundeszentrale für gesundheitliche Aufklärung (2007) Alkoholkonsum der Jugendlichen in Deutschland 2004 bis 2007. Ergebnisse der Repräsentativbefragungen der Bundeszentrale für gesundheitliche Aufklärung. Kurzbericht, Köln

8. Papst A, Kraus L (2008) Alkoholkonsum, alkoholbezogene Störungen und Trends. Ergebnisse des Epidemiologischen Suchtsurveys 2006. SUCHT 54(Sonderheft 1):36-46

9. Bundeszentrale für gesundheitliche Aufklärung (2008) Die Drogenaffinität Jugendlicher in der Bundesrepublik Deutschland 2008. Erste Ergebnisse zu aktuellen Entwicklungen und Trends. Köln

10. Deutsche Hauptstelle für Suchtfragen (Hrsg) (2009) Jahrbuch Sucht 2009. NEULAND Verlagsgesellschaft, Geesthacht

11. Kurth BM, Kamtsiuris $P$, Hölling $H$ et al (2008) The challenge of comprehensively mapping children's health in a nation-wide health survey: design of the German KiGGS-Study. BMC Public Health (8):196

12. Hölling $H$, Kamtsiuris $P$, Lange $M$ et al (2007) Der Kinder- und Jugendgesundheitssurvey (KiGGS): Studienmanagement und Durchführung der Feldarbeit. Bundesgesundheitsbl Gesundheitsforsch Gesundheitsschutz 50:557-566

13. Kamtsiuris $P$, Lange $M$, Schaffrath Rosario A (2007) Der Kinder- und Jugendgesundheitssurvey (KiGGS): Stichprobendesign, Response und Nonresponse-Analyse. Bundesgesundheitsbl Gesundheitsforsch Gesundheitsschutz 50:547-556

14. Kurth BM (2007) Der Kinder- und Jugendgesundheitssurvey (KiGGS): Ein Überblick über Planung, Durchführung und Ergebnisse unter Berücksichtigung von Aspekten eines Qualitätsmanagements. Bundesgesundheitsbl Gesundheitsforsch Gesundheitsschutz 50:533-547

15. Mensink GBM, Bauch A, Vohmann C et al (2007) EsKiMo. Bundesgesundheitsbl Gesundheitsforsch Gesundheitsschutz 50:902-908 
16. Mensink GBM, Haftenberger M, Thamm M (2001) Validity of DISHES 98, a computerised dietary history interview: energy and macronutrient intake. Eur J Clin Nutr 55:409-417

17. Klemm C, Mathis G, Christ M et al (1999) Der Bundeslebensmittelschlüssel (BLS II.3). Bundesinstitut für gesundheitlichen Verbraucherschutz und Veterinärmedizin, Berlin

18. Lange $M$, Kamtsiuris $P$, Lange $C$ et al (2007) Der Kinder- und Jugendgesundheitssurvey (KiGGS): Messung soziodemographischer Merkmale im Kinder- und Jugendgesundheitssurvey und ihre Bedeutung am Beispiel der Einschätzung des allgemeinen Gesundheitszustands. Bundesgesundheitsbl Gesundheitsforsch Gesundheitsschutz 50:578-589

19. Lampert T, Heizmann B, Hagen C (2008) Gesundheitliche Ungleichheit bei Kindern und Jugendlichen in Deutschland. Robert Koch-Institut, Berlin

20. Burger M, Brönstrup A, Pietrzik (2000) Alkoholkonsum und Krankheiten. Schriftenreihe des Bundesministeriums für Gesundheit Bd. 134, Nomos, Baden-Baden

21. Seitz H, Bühringer G (2008) Empfehlungen des wissenschaftlichen Kuratoriums der DHS zu Grenzwerten für den Konsum alkoholischer Getränke. Deutsche Hauptstelle für Suchtfragen, Hamm (http://www.dhs.de)

22. Currie C, Gabhainn SN, Godeau E et al (Hrsg) (2008) Inequalities in young people's health: HBSC international report from the 2005/2006 survey. World Health Organization, Copenhagen

23. Kraus L, Pabst A, Steiner S (2008) Die Europäische Schülerstudie zu Alkohol und anderen Drogen 2007 (ESPAD): Befragung von Schülerinnen und Schülern der 9. und 10. Klasse in Bayern, Berlin, Brandenburg, Hessen, Mecklenburg-Vorpommern, Saarland und Thüringen. IFT Berichte 165. Institut für Therapieforschung, München

24. Kraus L, Heppekausen K, Barrera A, Orth B (2004) Die Europäische Schülerstudie zu Alkohol und anderen Drogen (ESPAD): Befragung von Schülerinnen und Schülern der 9. und 10. Klasse in Bayern, Berlin, Brandenburg, Hessen, MecklenburgVorpommern und Thüringen. IFT-Berichte 141. Institut für Therapieforschung, München

25. Richter M, Settertobulte W, Ravens-Sieberer U, Hurrelmann K (2007) Prävalenzen und Trends im Tabak- und Alkoholkonsum bei 11- bis 15-Jährigen: Ergebnisse der nordrhein-westfälischen $\mathrm{HB}$ SC Studien 1994 bis 2006. In: Wichmann HE, Nowak D, Zapf A (Hrsg) Kongress Medizin und Gesellschaft 2007, Augsburg 17-21. Abstractband Rheinware, Mönchengladbach, S 279-280

26. Bundeszentrale für gesundheitliche Aufklärung (2004) Die Drogenaffinität Jugendlicher in der Bundesrepublik Deutschland 2004, Teilband Alkohol. Köln

27. Anderson P, Baumberg B (2006) Alkohol in Europe. Institute of Alcohol Studies, London

28. Schenk L, Neuhauser H, Ellert U (2008) Kinder- und Jugendgesundheitssurvey (KiGGS) 2003-2006: Kinder und Jugendliche mit Migrationshintergrund in Deutschland. Beiträge zur Gesundheitsberichterstattung des Bundes, Robert Koch-Institut, Berlin

29. Richter M, Leppin A, Gabhainn SN (2006) The relationship between parenteral socio-economic status and episodes of drunkeness among adolescents: findings from a cross-national survey. BMC Public Health 6:289

30. Babor T, Caetano R, Casswell S et al (2005) Alkoho - kein gewöhnliches Konsumgut. Hogrefe, Göttingen
31. Drogen und Suchtrat (2008) Empfehlungen des Drogen- und Suchtrates an die Drogenbeauftragte der Bundesregierung für ein Nationales Aktionsprogramm zur Alkoholprävention (http://www. bmg.bund.de)

32. Robert Koch-Institut, Bundeszentrale für gesundheitliche Aufklärung (2008) Erkennen - Bewerten - Handeln: Zur Gesundheit von Kindern und Jugendlichen in Deutschland. Robert Koch-Institut, Berlin

33. Casswell S (2004) Alcohol brands in young peoples everyday lives. New developments in marketing. Alcohol \& Alcoholism 39(6):471-476

34. Ellickson PL, Collins RL, Hambarsoomians K, McCaffrey DF (2005) Does alcohol advertising promote adolescent drinking? Results from a longitudinal assessment. Addiction 100(2):235-246

35. Hanewinkel R, Tanski SE, Sargent JD (2007) Exposure to alcohol use in motion pictures and teen drinking in Germany. Int J Epidemiol 36(5):10681077 